\title{
DESAGREGAÇÃO ESPACIAL DE ESTIMATIVAS DE EVAPOTRANSPIRAÇÃO REAL OBTIDAS A PARTIR DO SENSOR MODIS
}

\author{
MORRIS SCHERER WARREN
}

\author{
Agência Nacional de Águas (ANA), Brasília, DF, Brasil \\ morris@ana.gov.br
}

Recebido Junho de 2012 - Aceito Setembro de 2012

\begin{abstract}
RESUMO
Estimativas de evapotranspiração obtidas por sensoriamento remoto têm sido utilizadas no balanço hídrico de bacias hidrográficas, definição da recarga de aquíferos e monitoramento de estiagem. Mas limitações existem em aplicações locais, tais como, manejo agrícola e modelagem hidrológica, em virtude da necessidade de uma maior frequência de imageamento ou de uma melhor resolução espacial dos sensores orbitais atualmente disponíveis. No presente artigo é proposto um procedimento para melhorar a resolução das estimativas de evapotranspiração do sensor Moderate Resolution Imaging Spectroradiometer (MODIS), de $1 \mathrm{~km}$ para 500 metros. Esse procedimento é baseado na desagregação da banda termal do MODIS anteriormente ao cálculo da evapotranspiração. A comparação com o sensor ASTER permite constatar que é possível desagregar as estimativas do MODIS, com erro médio inferior a $25 \mathrm{~W} \mathrm{~m}^{-2}$ e correlação de 0,95 .
\end{abstract}

Palavras-chave: termal, sensoriamento remoto, modelos hidrológicos

\begin{abstract}
SPATIAL DISAGGREGATION OF ACTUAL EVAPOTRANSPIRATION ESTIMATIONS OBTAINED FROM MODIS SENSOR

Remotely sensed evapotranspiration estimations have been used for the calculation of the water balance in hydrographical basins, the definition of aquifer recharge, and the monitoring of drought events. However, there are limitations on local scale applications, such as agricultural management and hydrological modeling that arise from the lack of spatial resolution or the low frequency of image acquisition of current polar satellite constellations. The present article puts forward a procedure to improve the spatial resolution of evapotranspiration estimations obtained from the Moderate Resolution Imaging Spectroradiometer (MODIS), from $1 \mathrm{~km}$ to 500 meters resolution. The procedure is based on the disaggregation of the thermal band before estimating of evapotranspiration. Comparison with the ASTER sensor, at 500 meters resolution, indicates a mean error of $25 \mathrm{~W} \mathrm{~m}^{-2}$ and a correlation coefficient of 0.95 .
\end{abstract}

Keywords: thermal, remote sensing, hydrological models

\section{INTRODUÇÃO}

Em agrometeorologia experimentos de campo como fluxos turbulentos, razão de Bowen e cintilômetros são capazes de monitorar de forma precisa a evapotranspiração real em locais selecionados, mas a variabilidade da evapotranspiração é significativa e o balanço de energia pode levar à estimativas errôneas quando extrapolado regionalmente (Teixeira, 2010). De forma alternativa, modelos hidrológicos distribuídos são capazes de simular o fluxo de água no sistema solo-planta-atmosfera em áreas extensas (Raghunat et al., 1998; Panday e Huyakorn,
2004; DHI, 2008; Reichle, 2008). Estes modelos, entretanto, apresentam um alto nível de incerteza ligada à parametrização dos processos hidrológicos terrestres, como a evapotranspiração e a redistribuição de água no solo.

Inúmeras técnicas têm sido desenvolvidas para entender, quantificar e reduzir a incerteza em modelos hidrológicos utilizando observações hidrológicas distribuídas espacialmente (Madsen, 2003; Vieux, 2004; Beven e Biley, 1992; Walker e Houser, 2005; Vrugt et al., 2005; Gourley e Vieux, 2006). A utilização de sensoriamento remoto representa uma alternativa interessante para a obtenção do padrão espacial de 
variáveis hidrológicas (Grayson e Blöschl, 2000), sendo que o sensoriamento remoto é, em muitos casos, a única alternativa existente para a obtenção direta de estimativas distribuídas, como a evapotranspiração real.

A vantagem de utilizar o sensoriamento remoto para obter a evapotranspiração, em detrimento de outras perspectivas, como as baseadas na equação de Penman-Monteith em estações micrometeorológicas, é que sua estimativa pode ser obtida exclusivamente por meio da equação de balanço de energia, não sendo necessário se conhecer dados acerca da cobertura vegetal (ex. coeficiente da cultura ou resistência aerodinâmica) e do teor de água no solo (ex. coeficiente de estresse hídrico ou resistência superficial).

Em diferentes aplicações agrometeorológicas é desejável o uso de estimativas de evapotranspiração, com alta frequência temporal e resolução espacial, para se estabelecer uma relação biunívoca entre a medida de evapotranspiração, obtida da imagem de satélite, e um determinado polígono de uso da terra (ex. pivô de irrigação). A alta frequência temporal é necessária para se capturar a dinâmica da evapotranspiração ao longo do tempo, já que essa sofre alteração em função da quantidade de água precipitada ou aplicada por irrigação sobre o solo (teor de água no solo), além de ser influenciada por fatores micrometerológicos e fisionômicos/fisiológicos da vegetação (Allen et al., 1998).

$\mathrm{Na}$ atual geração de satélites, entretanto, as opções de sensores termais necessários para o cálculo da evapotranspiração real são restritas em relação à frequência de imageamento e a resolução espacial, representando limitações em aplicações de escala local, tais como, manejo agrícola e modelagem hidrológica. As opções atuais são de sensores termais de média resolução espacial e baixa resolução temporal (ETM, TM, ASTER); ou sensores termais de baixa resolução espacial e alta resolução temporal (MODIS, AVHRR).

Para contornar este problema, inúmeras técnicas têm sido propostas para a construção de séries temporais de evapotranspiração a partir do uso de satélites. Em geral, se pode agrupar essas técnicas em dois grupos: 1) técnicas de interpolação temporal das estimativas de evapotranspiração feitas a partir de sensores termais de média resolução espacial e baixa frequência temporal (Bastiaanssen et al., 1998; Allen et al., 2007); 2) técnicas de desagregação espacial das estimativas de evapotranspiração feitas a partir de sensores termais de baixa resolução espacial e alta frequência temporal (Chemin e Alexandridis, 2004; Kustas et al., 2003; Vazifedoust, 2007).

O primeiro grupo apresenta limitações em função da intermitência de eventos de precipitação e irrigação no período de aquisição de duas imagens termais de alta resolução espacial ( $\geq 16$ dias), no qual o padrão espaço-temporal da precipitação/irrigação altera a evapotranspiração em intervalo bastante inferior a 16 dias. Já no segundo grupo, a obtenção da evapotranspiração em baixa resolução espacial ocasiona erro, pois a agregação da evapotranspiração em diferentes escalas espaciais não é linear (Moran et al., 1997; Su et al., 1999; Kustas et al., 2003; McCabe e Wood, 2006; Li et al., 2008).

Desta forma, é proposto um novo procedimento para melhorar a resolução espacial das estimativas de evapotranspiração obtidas por satélite. Esse procedimento foi desenvolvido para atender os seguintes critérios: 1) usar o sensor Moderate Resolution Imaging Spectroradiometer (MODIS), o que resultará, potencialmente, em duas estimativas de evapotranspiração real por dia; 2) desagregar a temperatura do MODIS anteriormente à estimativa da evapotranspiração, evitando-se, assim, a não linearidade da evapotranspiração devido à heterogeneidade da superfície no pixel da imagem. O objetivo do presente artigo é apresentar um procedimento metodológico para obter estimativas de evapotranspiração com 500 de resolução usando o sensor MODIS, bem como analisar o procedimento por meio de validação cruzada com o sensor ASTER.

\section{MATERIAL E MÉTODOS}

\section{1 Área de estudo}

A área de estudo engloba a Bacia do Rio Preto dentro dos limites do Distrito Federal, estendendo-se à oeste em direção a Bacia São Bartolomeu, à leste na porção da Bacia do Rio Preto situada no município de Formosa - GO, ao sul englobando a parte superior da Bacia do Rio São Marcos e, ao norte, abrangendo as cabeceiras das bacias São Bartolomeu e Rio Preto (Figura 1). O uso do solo na área de estudo é basicamente agropecuário, com pouca ocorrência de áreas de Cerrado e matas galerias. Destaca-se na bacia a agricultura irrigada, responsável por cerca de $80 \%$ da produção agrícola do Distrito Federal, sendo realizada a irrigação por pivô central com água captada diretamente dos cursos d'água ou pequenas barragens (Rodrigues et al., 2007). Áreas de Cerrado podem ser encontradas à leste (porção da Bacia do Rio Preto localizada em Formosa - GO) e à oeste da área de estudo (Bacia do Rio São Bartolomeu).

\subsection{Estimativa da evapotranspiração real com resolução espacial de 500 metros}

No presente artigo a evapotranspiração foi estimada na resolução espacial de 500 metros usando o sensor MODIS. A estimativa e a avaliação da evapotranspiração foram executadas em três etapas: 1. desagregação espacial da banda termal do MODIS ; 2. aplicação do modelo Mapping EvapoTranspiration 


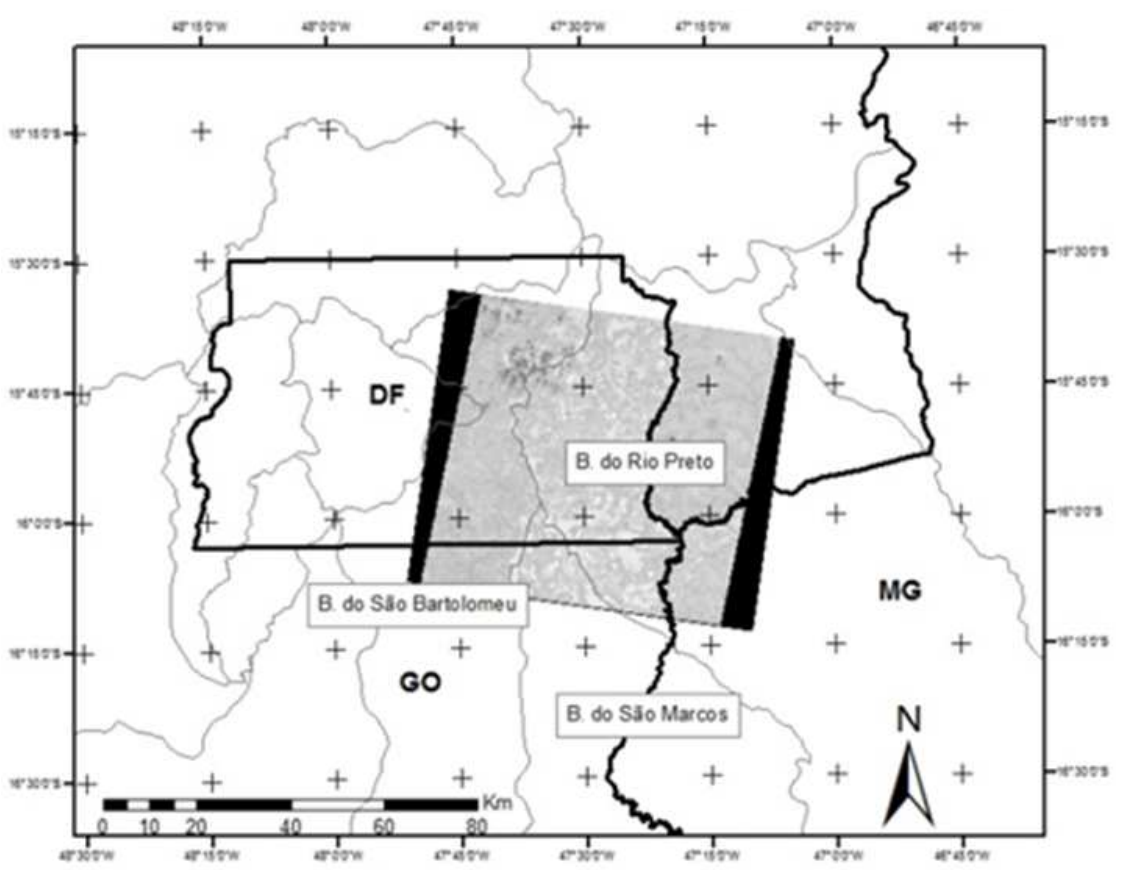

Figura 1 - Imagem ASTER selecionada para a validação da desagregação espacial da evapotranspiração obtida do MODIS.

at high Resolution with Internalized Calibration -METRIC (Allen et al., 2007); 3. validação cruzada usando como referência o sensor ASTER.

\subsubsection{Desagregação espacial da banda termal do MODIS}

A melhoria da resolução espacial da banda termal tem sido feita por meio de técnicas estatísticas e de análise espacial, genericamente denominadas em inglês de "thermal sharpening". No presente artigo, o termo desagregaré adotado como equivalente ao termo inglês sharpening, ou o procedimento para melhorar a resolução espacial de uma superfície numérica qualquer.

Para realizar a desagregação da temperatura foram utilizados um parâmetro de vegetação e duas variáveis sensíveis à umidade superficial, respectivamente, o Índice de Vegetação por Diferença Normalizada - NDVI, o Índice de Umidade por Diferença Normalizada - NDWI (Gao, 1996) e a componente Wetnness da transformação Tasseled Cap - TCW (Kauth e Thomas, 1976; Crist e Cicone, 1984).

O algoritmo utilizado na desagregação da temperatura foi baseado em estimativa por "mínimos quadrados", sendo inspirado no algoritmo de Liu e Pu (2008) para melhoria da resolução espacial da radiância termal usando dados de emissividade da superfície. Inicialmente foi realizada a regressão linear entre a temperatura da imagem de baixa resolução $(1 \mathrm{~km})$ e os parâmetros-variáveis auxiliares NDVI, NDWI e TCW, com resolução espacial de aproximadamente 500 metros (Figura 2). Esta estimativa foi obtida por mínimos quadrados considerando toda a imagem, ou seja, trata-se de uma estimativa global para a área de estudo.

A esta estimativa inicial (temperatura correspondente à resolução de 500 metros) foi imposta uma restrição de preservação dos valores de temperatura da menor resolução (1 km), ou seja, o valor de temperatura observado na imagem de baixa resolução espacial pode ser novamente obtido ao realizar a média da temperatura da imagem desagregada. Isto foi realizado em três etapas: 1) inicialmente obteve-se a média da estimativa da temperatura de todos os sub-pixels dentro do pixel de $1 \mathrm{~km}$; 2) o valor de temperatura observado na resolução de $1 \mathrm{~km}$ (imagem original) foi subtraído da estimativa de temperatura agregada na etapa $1 ; 3$ ) a diferença obtida na etapa 2 (positiva ou negativa) foi adicionada a cada um dos sub-pixel. Esta estimativa da temperatura normalizada (temperatura estimada de 500 metros com adição de erro) substituiu a imagem de temperatura original de $1 \mathrm{~km}$. O procedimento se repetiu de forma iterativa até o ponto em que não houve incremento adicional no valor de correlação com os parâmetros e/ou variáveis auxiliares.

\subsubsection{Evapotranspiração real usando o sensor MODIS}

A evapotranspiração real foi calculada, a partir da banda termal desagregada para 500 metros, usando o modelo METRIC (Allen et al., 2007). O fluxo de calor latente ( $\mathrm{W} \mathrm{m}^{-2}$ ) no METRIC, que representa a energia utilizada no processo de evapotranspiração, é obtido pela equação de balanço de energia 


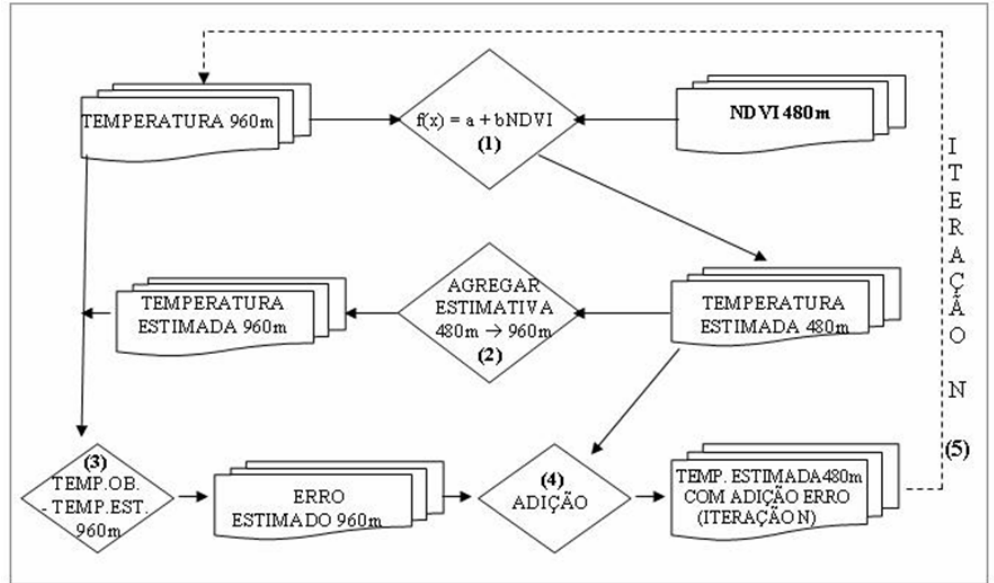

Figura 2 - Fluxograma de processamento do algoritmo Global de desagregação da banda.

uma vez conhecidos o fluxo de calor sensível, o fluxo de calor no solo e o saldo de radiação:

$$
\mathrm{LE}=\mathrm{R}_{\mathrm{n}}-\mathrm{G}_{0}-\mathrm{H}
$$

em que $\mathrm{LE}=$ fluxo de calor latente $\left(\mathrm{W} \mathrm{m}^{-2}\right) ; \mathrm{R}_{\mathrm{n}}=$ saldo de radiação $\left(\mathrm{W} \mathrm{m}^{-2}\right) ; \mathrm{G}=$ fluxo de calor no solo $\left(\mathrm{W} \mathrm{m}^{-2}\right) ; \mathrm{H}=$ fluxo de calor sensível $\left(\mathrm{W} \mathrm{m}^{-2}\right)$.

Para estimar o fluxo de calor sensível, o METRIC utiliza o gradiente de temperatura (dT) em detrimento da temperatura radiométrica. $\mathrm{O}$ gradiente de temperatura próximo a superfície (dT) é obtido por meio de uma indexação linear com a temperatura superficial $\left(\mathrm{T}_{\mathrm{s}}\right)$ :

$$
\mathrm{dT}=\mathrm{a}+\mathrm{bT}_{\mathrm{s}}
$$

Os coeficientes "a" e "b" são obtidos para cada imagem tomando-se como base dois pixels em situações extremas em termos da partição de energia, no qual dT pode ser estimado: "pixel frio" e "pixel quente". O "pixel frio" é selecionado em local com vegetação em pleno desenvolvimento e sem restrição de umidade (alto valor do índice de vegetação e baixa temperatura superficial). Nesse pixel assume-se que a evapotranspiração ocorre um pouco acima da evapotranspiração de referência $\left(\mathrm{ET}_{\mathrm{a}}=1,05 \mathrm{ET}_{\text {ref }}\right)$. Esse valor $5 \%$ maior do que a evapotranspiração de referência foi observado em algumas áreas agrícolas em Idaho -EUA, local onde Allen et al. (2007) desenvolveram o METRIC, ou seja, algumas culturas agrícolas possuem evapotranspiração máxima 5\% superior a evapotranspiração de referência medida em uma estação meteorológica, situada sobre grama ou alfafa. Para a seleção do "pixel quente no METRIC, um balanço hídrico simplificado é executado para verificar se a evaporação é nula ou fornecer um valor de evaporação residual $\left(H=\left(R_{n}-G\right)-L_{\text {residual }}\right)$.

A partir desse ponto, se conhece dois pares da temperatura radiométrica e do gradiente de temperatura na imagem de satélite
$\left(\mathrm{dT}_{\text {quente }} \mathrm{e} \mathrm{T}_{\text {quente }} ; \mathrm{dT}_{\text {frio }} \mathrm{e} \mathrm{T}_{\text {frio }}\right)$. Uma regressão linear simples é utilizada para se obter o intercepto e o coeficiente angular da regressão entre o gradiente de temperatura e a temperatura superficial ("a" e "b" da Equação 2). A Equação 2 é então aplicada a todos os pixels da imagem, resultando no gradiente de temperatura e, pela aplicação da Equação 3, em uma primeira estimativa do fluxo de calor sensível.

$$
\mathrm{H}=\rho_{\mathrm{ar}} \mathrm{C}_{\mathrm{p}} \frac{\mathrm{dT}}{\mathrm{r}_{\mathrm{ah}}}
$$

em que $\rho_{\mathrm{ar}}=$ densidade do ar $\left(\mathrm{kg} \mathrm{m}^{-3}\right) ; \mathrm{C}_{\mathrm{p}}=$ calor específico do ar em pressão constante $\left(\mathrm{J} \mathrm{kg}^{-1} \mathrm{~K}^{-1}\right)$ e $\mathrm{r}_{\text {ah }}=$ resistência aerodinâmica ao fluxo de calor sensível $\left(\mathrm{s} \mathrm{m}^{-1}\right), \mathrm{dT}=$ gradiente de temperatura próximo a superfície (K), de forma aproximada, dT corresponde a diferença de temperatura entre a superfície e a atmosfera adjacente, promovendo o fluxo de calor sensível.

Deste ponto em diante ocorre um processo iterativo para ajustar $\mathrm{H}$ em função da instabilidade atmosférica, no qual a cada iteração os valores da resistência aerodinâmica $\left(\mathrm{r}_{\mathrm{ah}}\right)$, do gradiente de temperatura (dT), da velocidade de atrito $\left(\mathrm{u}_{*}\right)$ são atualizados usando a formulação de Monin-Obukhov para considerar as condições de instabilidade atmosférica (Allen et al, 2007).

Em relação à proposta original de Allen et al. (2007), as únicas alterações foram a utilização dos produtos de temperatura e radiância do MODIS. Os produtos MODIS utilizados foram o MOD11-L2 e o MOD01HKM. O MOD11-L2 é o produto de temperatura/emissividade gerado diariamente com resolução de $1 \mathrm{~km}$ a partir da aplicação do algoritmo "Split Window" (Wan e Dozier, 1996). O MOD01HKM é o produto diário de radiância corrigida, tendo resolução espacial de 500 metros. Nesse último caso, os valores de radiância foram transformados para reflectância de superfície e albedo de acordo com a proposta de Tasumi et al. (2008). 


\subsubsection{Validação cruzada usando o ASTER}

A fim de analisar a qualidade da estimativa de evapotranspiração do MODIS com 500 metros de resolução, todos os componentes do balanço de energia do MODIS e do ASTER foram comparados na resolução de 500 metros.

Para o sensor ASTER, o balanço de energia foi também calculado por meio do modelo METRIC (Allen et al., 2007), sendo a estimativa inicialmente feita na resolução de 90 metros e posteriormente degradado para $\sim 500$ metros considerando explicitamente a função de espalhamento pontual do MODIS. Para o cálculo do saldo de radiação do ASTER foi empregada a proposta de Allen et al. (2007), sendo o albedo calculado conforme Wubet (2003), a temperatura obtida do produto AST08 (Gillespie et al., 1998), as emissividades em banda estreita das bandas termais do ASTER obtidas do produto AST05 (Gillespie et al., 1998) e a emissividade em banda larga estimada com os pesos derivados por Ogawa et al. (2002).

Esta comparação foi realizada em uma área situada a leste do Distrito Federal, correspondente a sobreposição das imagens ASTER e MODIS obtidas simultaneamente no dia 7 de maio de 2000 (Figura 1).

\section{RESULTADOS E DISCUSSÃO}

\subsection{Temperatura}

A diferença média entre os produtos MOD11L2 e AST08 na resolução de $1 \mathrm{~km}$ é de $2,71 \mathrm{~K}$. Ao desagregar esta estimativa para 500 metros, a diferença média aumenta para $2,72 \mathrm{~K}$, ou seja, semelhante à resolução de $1 \mathrm{~km}$. Esta discrepância ocorre devido às diferenças entre os algoritmos AST08 e MOD11-L2, sem necessariamente interpretar que o valor subestimado da temperatura desagregada seja resultante do algoritmo de desagregação. $\mathrm{O}$ coeficiente de correlação encontrado entre a temperatura do MODIS desagregada para 500 metros e a temperatura do ASTER (AST08) foi de 0,95 (Figura 3).

Nesta figura, destaca-se a superestimativa da temperatura do ASTER (AST08) comparativamente a temperatura do MODIS (MOD11). Na bibliografia consultada, trabalhos comparativos mostraram discrepâncias em torno de $+3 \mathrm{~K}$ a mais para o produto AST08 em relação ao MOD11 para áreas áridas e semiáridas (Liu et al., 2007; Merlin et al., 2010). Na presente análise, esta discrepância é confirmada, sendo a temperatura do AST08 cerca de 2,7 K superior a temperatura do MOD11 na resolução de 1 km (Figura 3).

Ao analisar a emissividade das bandas termais utilizadas no algoritmo "Split Window" do produto MOD11, constata-se valores médios muito altos, respectivamente 0,985 e 0,989 , para as bandas 31 e 32 do MODIS. Estes valores de emissividade

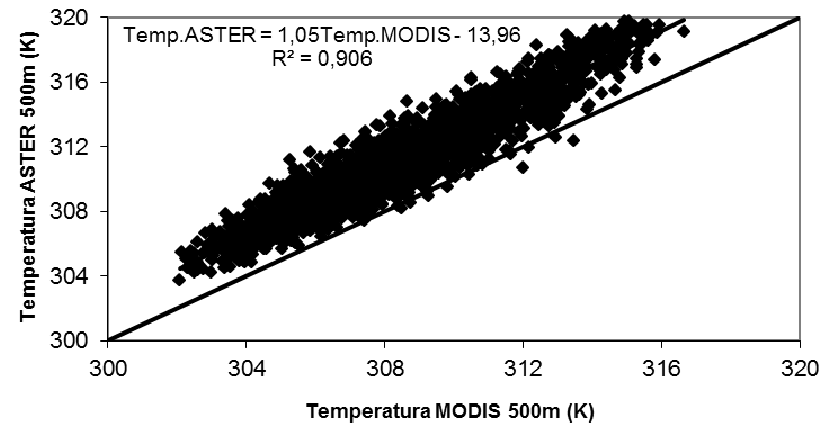

Figura 3 - Comparação AST08 x MOD11A1(desagregado): 500 m.

são muito próximos ao valor da emissividade da água pura (emissividade de $\sim 0,99$ ). Destaca-se, porém, que a imagem utilizada está em uma área essencialmente agrícola (Bacia do Rio Preto - DF e entorno), devendo possuir valores médios de emissividade inferiores. Esta sobrestimativa da emissividade ocasiona a depreciação da temperatura.

A emissividade em um determinado pixel do produto MOD11 é determinada por classificação, no qual o pixel é atribuído a uma das quatorze classes de uso da terra prédefinidas, sendo também considerados fatores dinâmicos, como a senescência da vegetação e o índice de vegetação (Snyder et al., 1998). Os autores admitem que um problema do método proposto é não considerar a umidade da superfície.

Para a imagem trabalhada, as correlações da emissividade com o índice de vegetação (Figura 4a) e o índice de umidade (Figura 4b) são nulas, o que corrobora a constatação de inadequação da estimativa de emissividade pelo método da classificação para o entorno de Brasília.

A emissividade do produto MOD11, calculada pelo método de classificação (Snyder et al., 1998), foi substituída pela emissividade calculada a partir do índice de área foliar (Allen et al., 2007) usando a seguinte fórmula (Warren et al., 2012):

$$
T_{s}=\frac{K_{2}}{\lambda \ln \left(\frac{K_{1} \varepsilon_{c}}{\lambda^{5} \varepsilon_{\text {MODIS1 } 1 c} B_{c}\left(T_{s_{-} \text {MODI1 }}\right)}+1\right)}
$$

em que Ts é a estimativa da temperatura superficial com uma nova emissividade $(\mathrm{K}) ; \lambda$ é o comprimento de onda médio das bandas 31 e 32 do MODIS ( $\mu \mathrm{m}) ; \mathrm{T}_{\mathrm{s}_{-} \text {MOD11 }}$ é a temperatura do produto MOD11 (K); K1 e K2 são constantes universais do sensor MODIS; $\varepsilon_{\text {MODIS11c }}$ é a emissividade média das bandas 31 e 32 do MODIS (adimensional); $\varepsilon_{\mathrm{c}}$ é a nova emissividade (adimensional), conforme proposta de Allen et al. (2007):

$$
\varepsilon_{\mathrm{C}}=\left\{\begin{array}{lr}
0,97+0,003 \times \mathrm{IAF} & \text { para IAF } \leq 3 \\
0,98 & \text { para IAF }>3
\end{array}\right.
$$

em que IAF é o índice de área foliar. 

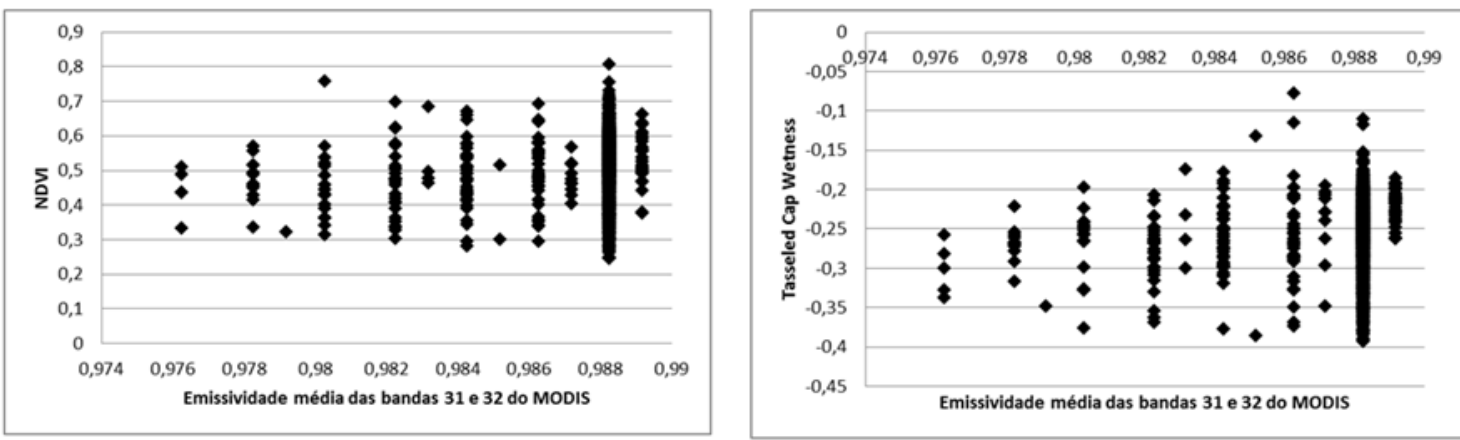

Figura 4 - Emissividade comparada com o NDVI (a) e com a TCW (b).

No presente artigo, o IAF foi correlacionado linearmente com o NDVI (Justice, 1986):

$$
\mathrm{IAF}=\mathrm{IAF}_{\max }\left[\frac{\left(\mathrm{NDVI}_{\mathrm{i}}-\mathrm{NDVI}_{\min }\right)}{\left(\mathrm{NDVI}_{\max }-\mathrm{NDVI}_{\text {min }}\right)}\right]
$$

em que $\mathrm{IAF}_{\max }$ é o índice de área foliar máximo da classe de uso da terra; NDVI é o índice de vegetação por diferença normalizada de cada pixel da imagem; $\mathrm{NDVI}_{\max }$ e $\mathrm{NDVI}_{\min }$ são os índices de vegetação mínimo e máximo para cada uso da terra.

Ao substituir a emissividade obtida por classificação (Snyder et al., 1998) pela emissividade calculada a partir do índice de área foliar (Allen et al., 2007), a diferença de temperatura entre o AST08 e o MOD11, na resolução de 500 metros, praticamente diminuiu $1 \mathrm{~K}$ (Figura 5), de 2,72 para $1,73 \mathrm{~K}$. A correlação não alterou ( 0,91 para ambas imagens) e o coeficiente angular se aproximou mais do valor unitário $(1,05$ para 1,04). A diferença relativa entre os produtos AST08 e MOD11 caiu 5\%, de 15 para $10 \%$ após substituir a emissividade.

\subsection{Saldo de radiação}

A temperatura superficial (item 3.1) entra no cálculo da radiação emitida em ondas longas (Rl $\uparrow)$, alterando, portanto, o valor do saldo de radiação $\left(\mathrm{R}_{\mathrm{n}}\right)$. Para calcular $\mathrm{R} l \uparrow$ é utilizada a

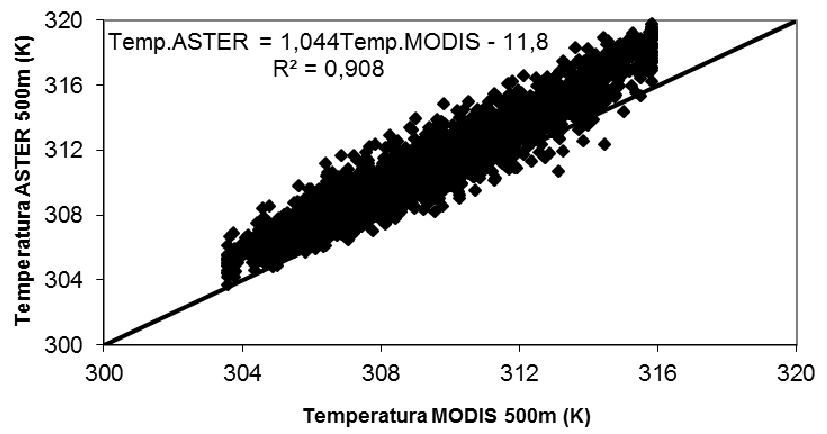

Figura 5 - Comparação AST08 x MOD11A1 (desagregado e emissividade ajustada): $500 \mathrm{~m}$. equação de Stefan-Boltzmann, sendo a temperatura elevada à quarta potência $\left(\mathrm{R} l \uparrow=\varepsilon_{0} \sigma \mathrm{T}_{\mathrm{s}}^{4}\right)$, usando a emissividade de banda larga entre 8 e $14 \mu \mathrm{m}$ (Allen et al., 2007). Isso implica que as diferenças de temperatura entre o MODIS e o ASTER são potencializadas ao se comparar Rl个. Além da temperatura da superfície, outra diferença presente na comparação de Rl $\uparrow$ é relativa à especificação da emissividade em banda larga utilizada no caso do ASTER e do MODIS. A Figura 7 mostra as diferenças de Rl $\uparrow$ entre o MODIS e o ASTER, considerando no cálculo a temperatura da superfície e a emissividade em banda larga. Os valores mais baixos de $\mathrm{Rl} \uparrow$ são semelhantes, sendo ampliadas as diferenças à medida que se consideram maiores valores de $\mathrm{R} 1 \uparrow$, em média $25 \mathrm{~W} \mathrm{~m}^{-2}$ maiores para o ASTER (Figura 6).

Esta diferença na parte superior da distribuição de $\mathrm{R} l \uparrow$ pode ser observada no cálculo do saldo de radiação (Figura 7). No cálculo de $R_{n}$, $R 1 \uparrow$ é subtraído, então uma subestimativa de $\mathrm{R} 1 \uparrow$ ocasiona uma superestimativa de $\mathrm{R}_{\mathrm{n}}$. A maior diferença de $\mathrm{R}_{\mathrm{n}}$ foi observada na parte inferior da distribuição (esquerda da Figura 8), correspondente às áreas mais quentes de solo exposto.

\subsection{Fluxo de calor sensível}

A Figura 8 mostra a diferença entre o fluxo de calor sensível (H) entre o MODIS e o ASTER. Na parte inferior

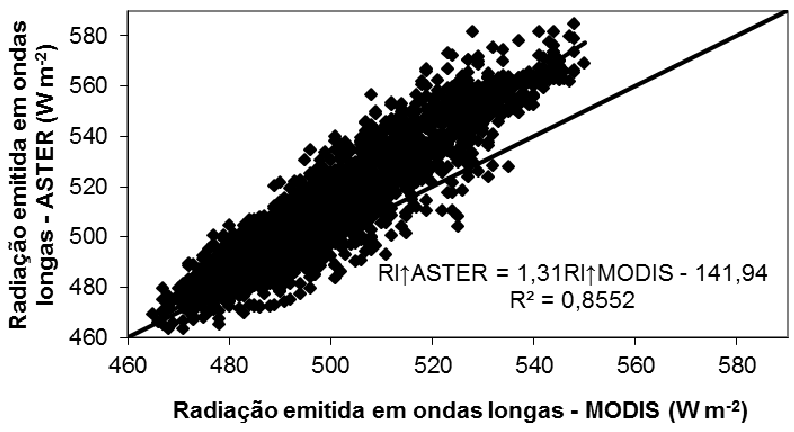

Figura 6 - Comparação da radiação emitida em ondas longas entre o MODIS e o ASTER. 


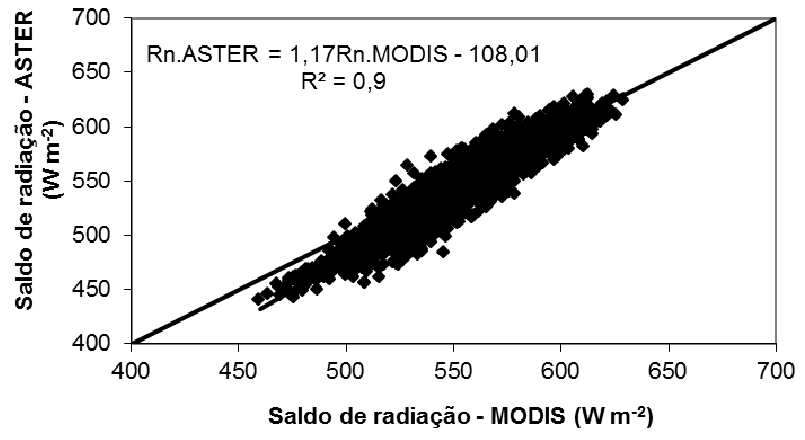

Figura 7 - Comparação do saldo de radiação entre o MODIS e o ASTER.

da distribuição H do ASTER é maior que H do MODIS, invertendo-se essa tendência na parte superior da distribuição. Basicamente dois fatores explicam essa discrepância:

1. A diferença de Rn. Conforme apresentado, as diferenças das estimativas da temperatura superficial e da emissividade em banda larga entre os dois sensores é propagada no cálculo de $\mathrm{Rl} \uparrow$ e Rn.

2. A diferença de escala na seleção dos pixels frio e quente. A melhor resolução do sensor ASTER (maior escala) ocasiona uma maior dispersão estatística dos valores de temperatura, ou seja, observa-se maior valor do pixel quente $(+4,2 \mathrm{~K})$ e menor valor de temperatura do pixel frio $(-2,5 \mathrm{~K})$.

\subsection{Fluxo de calor latente}

Ao calcular o fluxo de calor latente (LE), os desvios sistemáticos são atenuados entre o ASTER e o MODIS, a correlação é de 0,95 , com erro médio de $25 \mathrm{~W} / \mathrm{m}^{2}$ (Figura 9). Isso ocorre em virtude da internalização dos desvios de Rn e $\mathrm{G}$ no cálculo de $\mathrm{H}$. O modelo METRIC compensa uma série de incertezas (ligadas a correção atmosférica, temperatura superficial, albedo, resistência aerodinâmica, etc) introduzindo uma correção de desvio no cálculo de $\mathrm{H}$. Esse desvio introduzido

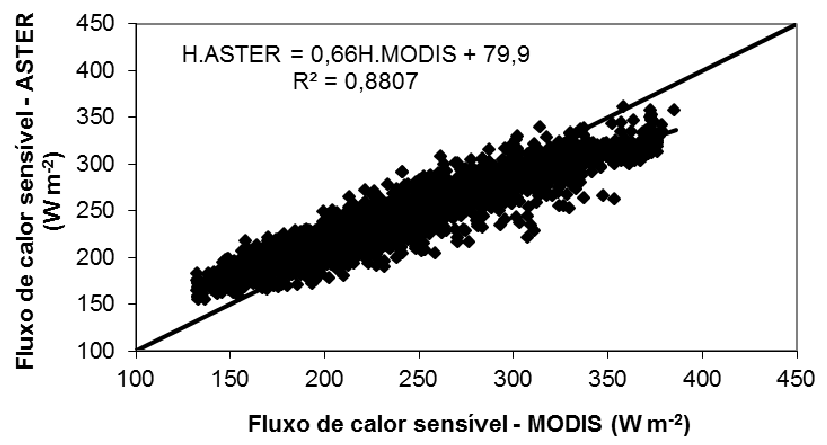

Figura 8 - Comparação do fluxo de calor sensível entre o MODIS e o ASTER. na estimativa de $\mathrm{H}$, fixando os valores de evapotranspiração do pixel frio e do pixel quente, compensa erros absolutos no cálculo de Rn e G, sendo posteriormente cancelados ao calcular o calor latente como "resíduo" da equação de balanço de energia para toda a imagem $(\mathrm{LE}=\mathrm{Rn}-\mathrm{G}-\mathrm{H})$. A amarra com os chamados "pixels âncoras" permite uma calibração interna das estimativas de evapotranspiração obtidas remotamente (Allen et al., 2007).

A superfície de LE resultante é, dessa forma, escalada de forma que os valores de evapotranspiração estejam entre zero e 1,05 da condição potencial, representando as situações de solo seco até de vegetação vigorosa e úmida. Essa suposição é adequada para a época seca na Bacia do Rio Preto - DF, no qual se pode observar áreas com forte estresse hídrico e áreas úmidas e bem vegetadas, tais como, pivôs de irrigação recém irrigados.

Deve-se também considerar que a estimativa de LE do sensor MODIS , mesmo com a temperatura desagregada para a resolução de 500 metros, ocorre sobre superfícies heterogêneas, ou seja, a resolução de 500 metros não garante a homogeneidade intra-pixel para toda a imagem. Na Figura 10 se pode observar pixels do MODIS (500 metros) com diferentes níveis de homogeneidade da superfície.

Ao selecionar apenas os pixels de maior homogeneidade da superfície, a correlação entre LE do ASTER e do MODIS aumentou de 0,95 para 0,98 , e o erro médio diminui de 25 para $10 \mathrm{~W} / \mathrm{m}^{2}$ (Figura 11). Dessa forma, ao desagregar espacialmente a evapotranspiração é importante observar a homogeneidade da superfície, ou seja, selecionar o pixel da imagem que esteja integralmente situado no interior da feição de interesse (ex. polígono de uso da terra).

A Figura 12 mostra a evolução temporal do coeficiente da cultura $(\mathrm{Kc})$ do feijão em cinco pivôs de irrigação na área de estudo. O Kc foi obtido pela razão entre a evapotranspiração remota e a evapotranspiração de referência medida em uma estação meteorológica em múltiplas datas. No primeiro mês e meio após o plantio nota-se valores de Kc entorno de 0,4 , com forte dispersão dos valores em relação a média. Essa grande dispersão dos valores está relacionada com a evaporação de

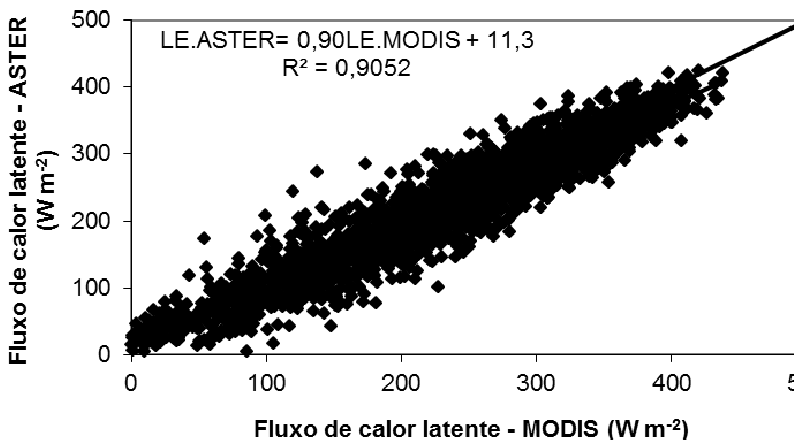

Figura 9 - Comparação do fluxo de calor latente entre o MODIS e o ASTER. 


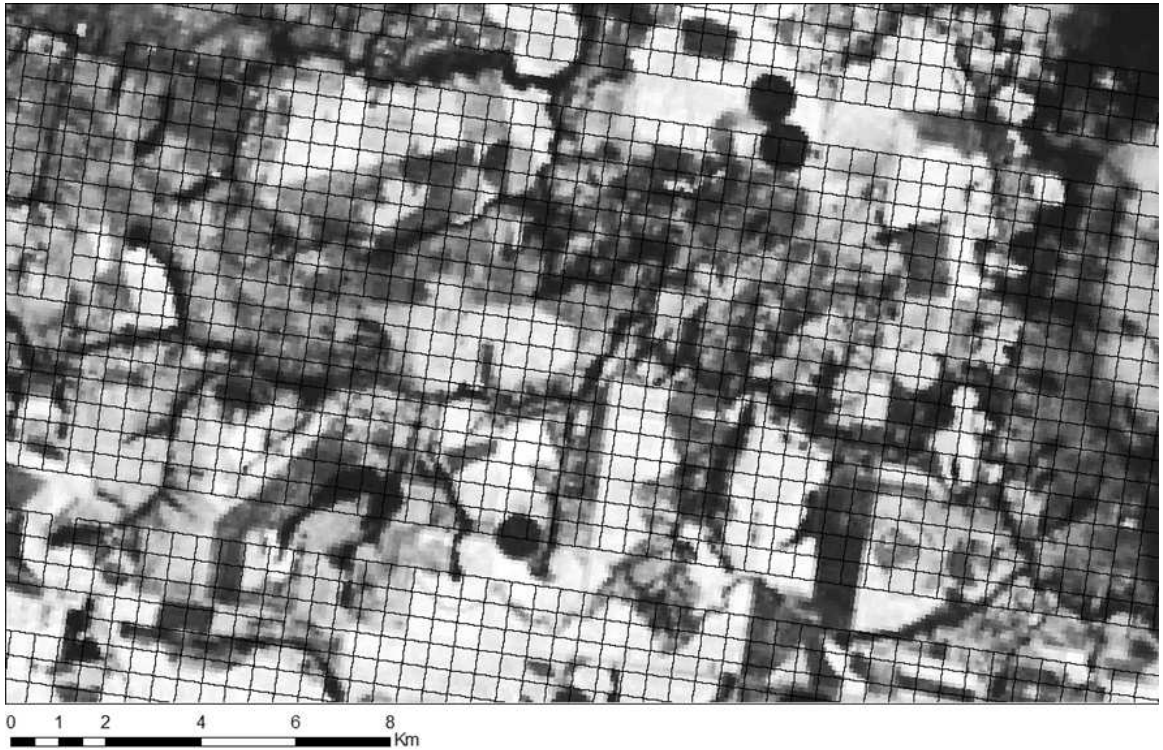

Figura 10 - Homogeneidade intra-pixel de cobertura do uso da terra na resolução de 500 metros.

água no solo no pivô de irrigação, apresentando forte oscilação em virtude das coincidências entre os dias de imageamento e presença/ausência de irrigação. Após a fase inicial (emergência da planta), percebe-se um crescimento rápido dos valores de Kc. O feijão atinge seu desenvolvimento pleno, cobrindo completamente a superfície do solo, e tornando a evaporação direta da água do solo secundária. A transpiração torna-se dominante e constante, atingindo valores um pouco acima e abaixo da evapotranspiração de referência. No final do período (setembro), a planta atinge o estágio de senescência ("amarelamento" das folhas), mas os impactos sobre os valores de evapotranspiração são menos evidentes, com diminuição pouco significativa dos valores de Kc.

\section{CONCLUSÕES}

Para a melhoria da resolução espacial das estimativas de evapotranspiração real do MODIS, foi inicialmente apresentado

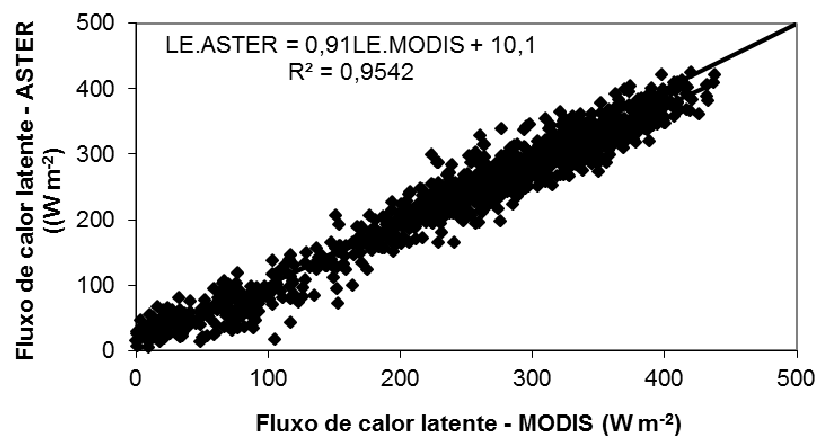

Figura 11 - Comparação do fluxo de calor latente entre o MODIS e o ASTER em pixel com homogeneidade de uso da terra. um algoritmo para a desagregação espacial da banda termal. A correlação entre as temperaturas superficiais obtidas pela MODIS (desagregado para 500 metros) e o ASTER (agregado para 500 metros) foi de 0,95 , mas a diferença média dos dois produtos foi de $2,71 \mathrm{~K}$, devido às diferenças entre os algoritmos para calcular a temperatura do MODIS e do ASTER.

Uma equação foi apresentada para alterar a emissividade do MOD11, permitindo diminuir a diferença entre o MOD11 e o AST08 em cerca de $1 \mathrm{~K}$. O refinamento da estimativa de temperatura é necessário, pois para calcular a radiação emitida em ondas longas, a temperatura é elevada a quarta potência, o que potencializa ainda mais qualquer desvio de estimativa. Essa discrepância se propaga e é perceptível no cálculo do saldo de radiação.

Para o cálculo do fluxo de calor latente, além das diferenças de temperatura, há as diferenças ligadas a seleção

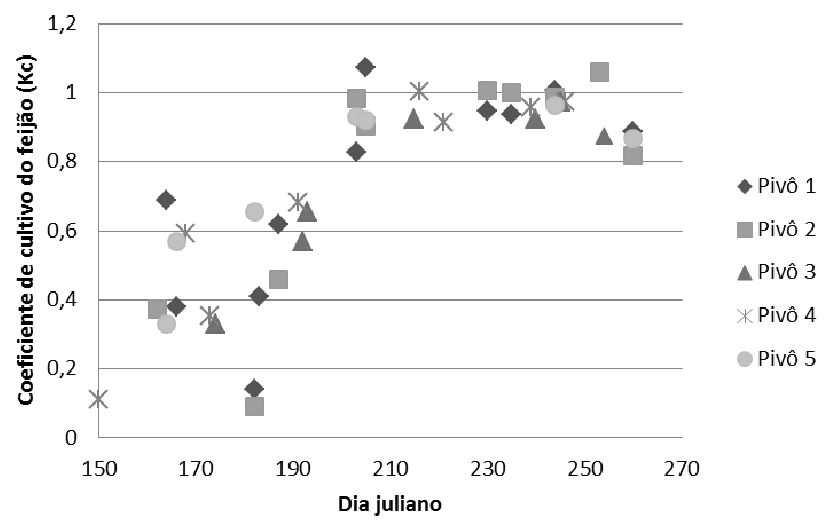

Figura 12 - Evolução do coeficiente de cultura - Kc do feijão obtido a partir de múltiplas imagens do MODIS. 
do pixel frio e quente em diferentes resoluções espaciais. No caso do ASTER, a seleção do pixel frio e quente foi realizada na resolução de 90 metros, o que ocasionou uma maior dispersão dos valores assumidos nesses dois pixels âncoras. Apesar de não realizada, uma análise de sensibilidade (múltiplas seleções dos pixels quente e frio) poderia ajudar a entender o impacto da seleção dos pixels âncoras nas estimativas de $\mathrm{H}$.

Por outro lado, o METRIC é capaz de internalizar essas incertezas no cálculo de $\mathrm{H}$, sendo posteriormente eliminadas ao se obter LE da equação de balanço de energia. A correlação de LE instantâneo entre o MODIS (desagregado para 500 metros) e o ASTER (agregado para 500 metros) foi de 0,95 . Ao selecionar apenas os pixels de maior homogeneidade da superfície, a correlação entre LE do ASTER e do MODIS aumentou de 0,95 para 0,98 .

A análise da evolução temporal do coeficiente de cultura do feijão em cinco pivôs de irrigação evidenciou o comportamento da evapotranspiração em relação aos diferentes estágios de desenvolvimento fenológico da planta, com contribuição variável da transpiração e da evaporação da água no solo. Esses resultados permitem constatar a viabilidade de utilização de estimativas de evapotranspiração desagregadas do MODIS, particularmente em aplicações locais que exijam alta frequência temporal, representando uma importante ferramenta em aplicações agro-meteorológicas.

Para a continuidade do trabalho, sugere-se a comparação das estimativas remotas diretamente com dados de evapotranspiração levantados em campo (ex. Razão de Bowen, ou Correlações Turbulentas ou Lisímetro). Neste caso, a validação seria baseada em dados coletados pontualmente (um pixel da imagem), mas pela utilização do sensor MODIS seria possível construir uma série temporal com múltiplas imagens. No caso de uma cultura temporária, seria possível realizar a validação do procedimento aqui proposto considerando diferentes estágios fenológicos da cultura agrícola e condições superficiais de umidade no solo.

\section{REFERÊNCIAS BIBLIOGRÁFICAS}

ALLEN, R.; PEREIRA, L.S.; RAES, D.; SMITH, M. Crop evapotranspiration: guidelines for computing crop water requirements. Roma: 1998, 300 p. (FAO Irrigation and Drainage Paper, v.56).

ALLEN, R.G.; TASUMI, M.; TREZZA, R. Satellite-based energy balance for mapping evapotranspiration with internalized calibration (METRIC) - model. Journal of Irrigation and Drainage Engineering, v.133, n.4, p.380-394, 2007.

BASTIAANSSEN, W. G. M.; MENENTI, M.; FEDDES, R. A.; HOLTSLAG, A. A. M. A remote sensing surface energy balance algorithm for land (SEBAL): 1. Formulation. Journal of Hydrology, v. 213, n. 1-4 , p. 198 -212, 1998.

BEVEN, K.; BILEY, A.M. The future of distributed models: model calibration and uncertainty prediction. Hydrological Processes, v.6, p.279-298, 1992.

CHEMIN, Y.; ALEXANDRIDIS, T. Improving spatial resolution of ET seasonal for irrigated rice in Zhanghe, China. Asian Journal of Geoinformatics, v.5, n. 1, p.311, 2004.

CRIST., E.P.; CICONE, R.C. A physically-based transformation of Thematic Mapper Data - the TM Tasseled Cap. Transactions on Geoscience and Remote Sensing, v. 41, p.2674-2677, 1984.

DHI - DANISH HYDRAULIC INSTITUTE. MIKE-SHE user manual. Estocolmo: DHI, 436 p., 2008.

GAO.B. NDWI. A normalized difference water index for remote sensing of vegetation liquid water from space. Remote Sensing of Environment, v.58, p.257-266, 1996.

GILlESPIE, A; ROKUGAWA, S., MATSUNAGA, T., COTHERN, J.S., HOOK, S.; KAHLE, A.B.. A Temperature and Emissivity Separation Algorithm for Advanced Spaceborne Thermal Emission and Reflection Radiometer (ASTER) Images. Transactions on Geoscience and Remote Sensing, v. 36, p.1113-1126, 1998.

GOURLEY, J.J.; VIEUX, B.E. A method for identifying sources of model uncertainty in rainfall-runoff simulations. Journal of Hydrology, v.327, p.68-80, 2006.

GRAYSON, R.B.; BLÖSCHL, G. Spatial patterns in catchment hydrology. Cambridge: Cambridge University Press, 1 ed., 404 p., 2000.

JUSTICE, C.O. Monitoring east African vegetation using AVHRR data. International Journal of Remote Sensing, v.6, p.1335-1372, 1986.

KAUTH, R.; THOMAS, G.S. The tasseled Cap - a graphic description of the spectral-temporal development of agricultural crops as seen by LANDSAT. LARS Symposia, artigo 159, 1976.

KUSTAS, W.P.; NORMAN, J.M.; ANDERSON, M;.C.; FRENCH, A.N. Estimating subpixel surface temperatures and energy fluxes from the vegetation index-radiometric temperature relationship. Remote Sensing of Environment, v.85, p.429-440, 2003.

LI, F.; KUSTAS, W.P.; ANDERSON, M.C.; PRUEGER, J.H.; SCOTT, R.L.. Effects of remote sensing spatial resolutions on interpreting tower-based flux observations. Remote Sensing of Environment, v.112, p.337-349, 2008.

LIU, D.; PU, R. Downscaling. Thermal Infrared Radiance for Subpixel Land Surface Temperature Retrieval. Sensors, v. 8, p.2695-2706, 2008. 
LIU, Y.; YAMAGUCHI, Y.; KE, C. Reducing the discrepancy between ASTER and MODIS land surface temperature products. Sensors, v.7, p.3043-3057, 2007.

MADSEN, H. Parameter estimation in distributed hydrological catchment modeling using automatic calibration with multiple objectives. Advances in Water Resources, v.26, p.205-216, 2003.

MACCABE, M.F.; WOOD, E.F. Scale influences on the remote estimation of evapotranspiration using multiple satellite sensors. Remote Sensing of Environment, v.105, p.271285,2006

MERLIN, O.; DUCHEMIN, B.; HAGOLLE, O.; JACOB, F.; COUDERT, B.; CHEHBOUNI, G.; DEDIEU, G.; GARATUZA, J.; KERR, Y. Disaggregation of MODIS surface temperature over an agricultural area using time series of Formosat-2 images. Remote Sensing of Environment, v.114, p.2500-2512, 2010.

MORAN, M.S.; HUMES, K.S., PINTER JR., P.J. The scaling characteristics of remotely-sensed variables for sparselyvegetated heterogeneous landscapes. Journal of Hydrology, v.190, p.337-362, 1997.

OGAWA, K.; SCHMUGGE, T.; JACOB, F.; FRENCH, A. Estimation of broadband land surface emissivity from multispectral thermal infrared remote sensing. Agronomie, v.22, p.695-696, 2002.

PANDAY, S.; HUYAKORN, P.S. A fully coupled physicallybased spatially-distributed model for evaluating surface/ subsurface flow. Advances in Water Resources, v.27, p.361-382, 2004.

RAGHUNATH, J.H.A.; HERATH, S.; MUSIAKE, K. Application of IIS distributed hydrological modelo (IISDHM) in Nakhon Sawn catchment, Thailand. Annual Journal of Hydraulic Engineering, v.42, 145-150 p., 1998.

RODRIGUES, L. N.; SANO, E. E.; AZEVEDO, J. A. de; SILVA, E. M. da. Distribuição espacial e área máxima do espelho d'água de pequenas barragens de terra na Bacia do Rio Preto. Espaço e Geografia, v. 10, p. 101-122, 2007.

REICHLE, R.H. Data assimilation methods in the earth sciences. Advances in Water Resources, v.31, n. 11, p.1411-1418, 2008.

SNYDER, W.C.; WAN, Z.; ZHANG, Y.; FENG, Y.-Z. Classification-based emissivity for land surface temperature measurement from space. International Journal of Remote Sensing, v.19, p.2753-2774, 1998.
SU, Z.; PELGRUM, H.; MENENTI, M. Aggregation effects of surface heterogeneity in land surface processes. Hydrology and Earth System Sciences, v.3, n. 4, p.549-563, 1999.

TASUMI, M.; ALLEN, R.G.; TREZZA, R. At-surface reflectance and albedo from satellite for operational calculation of land surface energy balance. Journal of Hydrologic Engineering, 2008, v.13, p.51-63, 2008.

TEIXEIRA, A. H. de C. Determining Regional Actual Evapotranspiration of Irrigated Crops and Natural Vegetation in the São Francisco River Basin (Brazil) Using Remote Sensing and Penman-Monteith Equation. Remote Sensing, v. 2, p. 1287-1319, 2010.

VAZIFEDOUST, M. Development of an agricultural drought assessment system: integration of agrohydrological modelling, remote sensing and geographical information. 2007. 147p. Tese (Doutorado em Ciência da geoinformação e observação terrestre). Wageningen University, Wageningen, 2007.

VIEX, B.E. Distributed hydrological modeling using GIS. Dordrecht: Kluwer Academic publishers ed, 2 ed., 289 p., 2004.

VRUGT, J.A.; et al. Improved treatment of uncertainty in hydrologic modeling: combining the strengths of global optimization and data assimilation. Water Resources Research, v.41, n.1, p., 2005.

WALKER, J. P.; HOUSER, P.R. Hydrologic data assimilation. In: ASWATHANARAYANA, U. (Org.). Advances in water science methodologies. Londres: Taylor \& Francis, ed.1, p.25-48, 2005.

WAN, Z.; DOZIER, J. A Generalized Split-Window Algorithm for Retrieving Land-Surface Temperature from Space. Transactions on Geoscience and Remote Sensing, v. 34, p.892-905, 1996.

WARREN, M.S.; PERES, L.; RODRIGUES, L.N.; CAMPANA, N.A. Procedimento para Alteração da Emissividade do Produto de Temperatura do MODIS - MOD11. Revista Brasileira de Cartografia, v. 64, p. 465-474, 2012.

WUBET, M.T. Estimation of absolute surface temperature by satellite remote sensing. 2003. 121p. Dissertação (Mestrado em Ciência da Geo-informação e Observação Terrestre) ITC, Utrecht, 2003. 